\section{Infección por SARS-CoV-2. Una nueva enfermedad endotelial trombo-inflamatoria}

\author{
FRANCISCO SAMANIEGO ${ }^{a}$, GUILLERMO CONTE ${ }^{1}$
}

\section{SARS-CoV-2 infection as a thrombo-inflammatory endothelial disease}

Coronavirus disease 19 is characterized by an intense inflammatory response and a high incidence of thrombotic events. Autopsy studies show severe endothelial injury associated with thrombosis of the alveolar capillaries of the lungs and other organs. In the pathophysiology of this disease endothelial injury and dysfunction, inflammation and thrombosis are key factors for the development of severe phenotypes. Therefore, we should consider this entity as a systemic endothelial disease in which an obstructive microvascular syndrome secondary to an intense thrombo-inflammatory response leads to acute respiratory insufficiency and multiorgan failure. Heparin is an excellent drug to treat Covid-19 patients due to its anticoagulant, anti-inflammatory, antiviral and endothelial effects. Clinical guidelines agree that the use of heparin thromboprophylaxis is a component of Covid-19 coagulopathy treatment, even though the dose and duration of treatments are not well defined.

(Rev Med Chile 2020; 148: 1467-1474)

Key words: Coronavirus; Disseminated Intravascular Coagulation; Thrombosis.

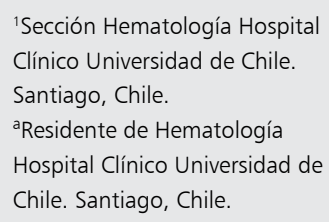

Trabajo no recibió financiamiento. Los autores declaran no tener conflictos de interés.

Recibido el 13 de julio de 2020 aceptado el 16 de septiembre de 2020 .

Correspondencia a:

Dr. Francisco Samaniego Hamburgo 800, Departamento 301, Ñuñoa. Santiago, Chile. francisco.samaniego.errazuriz@ gmail.com

Dr. Guillermo Conte gfconte@gmail.com
L a enfermedad por coronavirus 19 (Covid-19) causada por el coronavirus 2 del Síndrome Respiratorio Agudo Severo (SARS-CoV-2) fue reconocida inicialmente como una infección respiratoria con neumonía y síndrome de distrés respiratorio del adulto ${ }^{1}$. Sin embargo, es una enfermedad sistémica en la cual se produce un compromiso difuso del endotelio vascular y una intensa respuesta inflamatoria, siendo ambos factores responsables de producir una coagulopatía caracterizada por una elevada tendencia a la trombosis venosa, de la microcirculación y en menor medida arterial $^{2}$. La afección multiorgánica, con compromiso cardiovascular, respiratorio, neurológico, intestinal, hepático, pancreático, renal, cutáneo y hematológico, seria mediada por acción directa del virus, disfunción endotelial, inflamación y trombosis de la microcirculación de los órganos afectado $^{3}$. A nivel pulmonar, la trombosis masiva de la circulación capilar ${ }^{4}$, es parte esencial de los mecanismos fisiopatológicos responsables de la insuficiencia respiratoria y ha llevado a plantear el concepto de "síndrome microvascular obstructivo pulmonar" para esta entidad.

La coagulopatía inducida por Covid-19 (CIC) determina un elevado riesgo de tromboembolismo venoso (TEV) pese al uso de tromboprofilaxis farmacológica y es especialmente alto en pacientes que requieren Unidad de Cuidados Intensivos (UCI $)^{5,6}$. Esto ha llevado a usar dosis "intermedias" de heparina como tromboprofilaxis, sin embargo, no existe evidencia de alta calidad al respecto, por lo que las guías clínicas han formulado recomendaciones basadas en estudios observacionales, opinión de expertos y aplicando los principios sobre la prevención y tratamiento de la enfermedad tromboembólica en el paciente médico. 
Este trabajo tiene dos objetivos; 1) realizar una revisión comprensiva sobre los mecanismos fisiopatológicos que operan en la enfermedad por Covid-19 con énfasis en el rol central que tienen endotelio, inflamación y trombosis en la génesis de esta entidad y 2) revisión respecto a las recomendaciones sobre tromboprofilaxis en pacientes con Covid-19 y sus fundamentos.

\section{Endotelio, inflamación y trombosis en la enfermedad severa por SARS-CoV-2}

Estudios de autopsias sugieren el rol de la trombosis en la génesis de la falla ventilatoria por Covid-19. Pulmones de fallecidos por esta causa presentan trombosis vascular difusa con microangiopatía, daño endotelial y oclusión de capilares alveolares. También se ha descrito un proceso de angiogénesis prominente por intususcepción que altera la estructura capilar y que sería propio de esta entidad. Hallazgos relevantes adicionales son daño alveolar difuso con necrosis de neumocitos, edema y deposito intra alveolar de fibrina ${ }^{7-9}$. Dentro de todas estas alteraciones, la trombosis de la microcirculación pulmonar es uno de los mecanismos que explican la hipoxemia severa en estos pacientes $^{10}$.

El receptor utilizado por el virus para infectar las células del hospedero es la enzima convertidora de angiotensina 2 (ACE2). Esta es una metalopeptidasa expresada en múltiples sitios; vías respiratorias, pulmones, corazón, sistema nervioso, estomago, intestino, riñón, hígado y bazo. Su concentración es especialmente alta en el endotelio vascular, pulmones y corazón, lo que da cuenta del fenotipo más frecuente de la enfermedad ${ }^{11,12}$. Otra molécula que participaría en el ingreso del SARS-CoV-2 a la célula es la dipeptidil peptiasa 4 (DPP4). Esta es una enzima expresada en una gran cantidad de tejidos y su función principal es degradar incretinas involucradas en el metabolismo glucídico. Su expresión es mayor en el tejido adiposo y está asociada a insulinorresistencia, por lo que podría tener un rol en las formas severas de Covid-19 de pacientes diabéticos u obesos ${ }^{13}$. Pese a esto, en estudios en SARS CoV-1, su inhibición no ha demostrado evitar la infección celular por el virus ${ }^{14,15}$.

En los pulmones el SARS-CoV-2 infecta las células del epitelio alveolar y endotelio vascular.
Estas células, junto a macrófagos alveolares activados secretan citoquinas, quimoquinas y mediadores responsables del reclutamiento de leucocitos e inflamación ${ }^{16}$. La inflamación sistémica y la elevada concentración de citoquinas como IL-1, TNF alfa, IL-6 e IL-8, activan la coagulación por varios mecanismos y son esenciales en iniciar la $\mathrm{CIC}^{17-19}$. Monocitos y macrófagos activados expresan factor tisular y fosfatidil serina, neutrófilos liberan fibras de DNA e histonas conocidas como NETs (Neutrophil extracellular traps), células endoteliales activadas por el proceso inflamatorio y por efecto citopático directo del virus liberan multímeros de factor de Von Willebrand e inhibidores de la fibrinolisis como PAI-1 (inhibidor del activador del plasminógeno-1). Todos estos factores activan la agregación plaquetaria y el sistema de la coagulación llevando a la formación de trombos a nivel de los capilares alveolares determinando su oclusión $^{20}$. En condiciones normales, las citoquinas y leucocitos también participan en la degradación del trombo formado ${ }^{21}$. Esta estrecha interacción y regulación mutua entre inflamación y trombosis, se ha denominado "respuesta trombo-inflamatoria o inmuno-trombótica" y es clave en la fisiopatología de la infección por SARS-CoV-2 ${ }^{18,21}$.

El compromiso del endotelio vascular es esencial en la enfermedad Covid-19. El virus infecta el endotelio causando daño de éste, con infiltración por células inflamatorias, endotelitis y apoptosis. La severa disfunción endotelial resultante lleva a que el endotelio, normalmente con propiedades antiinflamatorias y antitrombóticas, cambie a un fenotipo proinflamatorio y protrombótico ${ }^{7,16}$. Esta afectación del endotelio es difusa y explica las anomalías vasculares y disfunción de los distintos órganos comprometidos ${ }^{22,23}$. Con esto se entiende que los factores de riesgo de padecer enfermedad severa sean aquellas condiciones asociados a disfunción endotelial, como son edad avanzada, obesidad, hipertensión arterial, diabetes y enfermedad cardiovascular ${ }^{3,24,25}$.

El compromiso multiorgánico, ya sea por infección directa de parénquimas o por cambios vasculares a nivel local está bien documentado. En una serie pacientes con distrés respiratorio por Covid-19 que cursaron con encefalopatía, se realizó resonancia magnética cerebral observándose hipoperfusión frontotemporal bilateral y accidentes encefálicos isquémicos ${ }^{26}$. Es interesante el reporte de casos de accidentes vasculares isquémicos en 
pacientes menores de 50 años y sin patología vascular previa $^{27}$ y el reporte de trombosis de la aorta en ausencia de placa de ateroma ${ }^{28}$ o pese a la terapia antiplaquetaria ${ }^{29}$. Esto refleja el severo compromiso vascular y estado protrombótico aun en áreas sin daño endotelial previo. A nivel cardiaco, los miocardiocitos expresan ACE2 siendo susceptibles de ser infectados por el SARS-CoV-2 resultando en miocarditis. Además, se describe una disfunción microvascular gatillada por citoquinas proinflamatorias y afectación de pericitos con consecuente isquemia miocárdica ${ }^{30}$. $\mathrm{Al}$ igual que en el endotelio pulmonar, se ha demostrado la presencia de partículas virales en el endotelio renal. Adicionalmente el virus SARS-CoV-2 puede infectar directamente células tubulares renales y podocitos a través de ACE2 y causar necrosis tubular aguda y glomerulopatía. Otros factores contribuyentes a la falla renal son la hipovolemia, la inflamación e hipoxemia ${ }^{31,32}$. En una serie de 412 pacientes con Covid-19, se observó, en 3\% de las tomografías abdominales realizadas, signos compatibles con isquemia intestinal. En los dos pacientes que requirieron resección intestinal se constató necrosis y trombos de fibrina en arteriolas submucosas, sugiriendo que la isquemia fue debida a la trombosis de pequeños vasos ${ }^{33}$. El páncreas endocrino puede estar afectado. Estudios in vitro demuestran que ACE2 es expresado en las células beta pancreáticas y que el SARS-CoV-2 es capaz de infectarlas. Además, el virus podría gatillar una respuesta inmune contra las células beta pancreáticas y favorecer el depósito de amiloide en estas. Finalmente, la intensa respuesta inflamatoria y el compromiso hepático contribuyen de forma adicional a alterar el metabolismo de la glucosa ${ }^{25,34}$.

Además de injuria vascular, en la enfermedad Covid-19 participan los restantes componentes de la triada de Virchow; anomalías del flujo sanguíneo e hipercoagulabilidad ${ }^{16}$. La hipercoagulabilidad se debe a un aumento de los niveles de fibrinógeno, factor VIII y factor Von Willebrand, gatillados por la respuesta inflamatoria y la disfunción endotelial. Adicionalmente hay aumento de factor tisular, secundario a la activación leucocitaria e hipoxia, aumento del dimero D (DD) y de PAI- ${ }^{16}$. Todo esto lleva a una activación de la coagulación y disminución de la fibrinolisis, con depósitos de fibrina a nivel de la microcirculación. Así, la CIC es un estado protrombótico y no hemorrágico siendo similar a la coagulopatía inducida por sepsis $(\mathrm{SIC})^{35}$. La coagulación intravascular diseminada (CID), en cambio, cursa con una coagulopatía de consumo e hiperfibrinólisis secundaria y es un estado hemorrágico. Es probable que la CIC y la CID sean parte de un continuo en el cual la CIC, en casos severos, puede progresar a una CID clásica. Esto explicaría la secuencia de cambios en los marcadores de la coagulación que ocurren en la enfermedad Covid-19, observándose inicialmente una elevación del fibrinógeno y del DD y más tardíamente, en los casos severos, una prolongación de los tiempos de la coagulación ${ }^{18}$. La elevación del DD podría ser un reflejo del intento frustro del sistema fibrinolítico de remover los trombos de fibrina de la microcirculación y los depósitos proteicos acumulados en alveolos, hipótesis que llevó a plantear el uso de plasminógeno o agentes fibrinolíticos en Covid-19. Series pequeñas muestran que el uso de plasminógeno nebulizado o agentes fibrinolíticos logra mejorar la oxigenación en estos pacientes. Sin embargo, el sistema fibrinolítico también podría contribuir con la infección viral. El virus puede activar la plasmina y usarla para clivar proteínas de su superficie permitiendo su ingreso a la célula blanco o para modular el sistema inmune gracias a efectos no enzimáticos de la plasmina sobre receptores de células inflamatorias. Estos efectos benéficos y deletéreos del sistema fibrinolítico, llamados la "paradoja de la plasmina", no ocurren de forma simultánea y plantean la posibilidad de usar agentes antifibrinolíticos en etapas tempranas de la infección para disminuir la infectividad del virus sin comprometer la eliminación de fibrina requerida en etapas más avanzadas de la enfermedad, donde habría beneficio de usar agentes fibrinolíticos ${ }^{36,37}$.

Considerando la fisiopatología de esta enfermedad, la heparina es el fármaco ideal gracias a su efecto anticoagulante, antiinflamatorio, antiviral y de protección endotelial. Similar a los proteoglicanos sulfatados endógenos, como el heparán sulfato, la heparina gracias a su estructura polisacárida sulfatada es capaz de unirse de forma inespecífica a una amplia gama de proteínas. De esta forma, se une a citoquinas, quimoquinas y proteínas del complemento neutralizándolos, interfiere con la quimiotaxis y migración de leucocitos, protege al endotelio de mediadores liberadas por células inflamatorias y activa vías de señalización de células endoteliales con efectos benéficos ${ }^{38}$. Adicionalmente, basado en el modelo de 
la "trombo-inflamación", la heparina disminuye la respuesta inflamatoria al bloquear la generación de trombina ${ }^{23}$. El efecto antiviral de las heparinas es de amplio espectro e incluye coronavirus, virus herpes, influenza y VIH. El anclaje del coronavirus a la célula blanco es facilitado por la interacción de las proteínas de la superficie viral con proteoglicanos celulares. La heparina se une a las proteínas de superficie del virus, incluyendo la proteína Spike, que es el ligando de ACE2, interfiriendo con la unión del virus a la célula blanco ${ }^{39,40}$.

\section{Tromboprofilaxis en pacientes hospitalizados por Covid-19}

De todos los eventos tromboembólicos, se estima que 50 a $60 \%$ ocurren en contexto de una hospitalización. Adicionalmente, los pacientes hospitalizados por Covid-19 tienen otros factores de riesgo de trombosis, como son edad avanzada, inmovilización, infección activa, insuficiencia respiratoria u obesidad, por lo que serían categorizados como de alto riesgo de trombosis y se recomendaría el uso de tromboprofilaxis farmacológica con heparina de bajo peso molecular (HBPM) por 6-14 días, en base a las guías de tromboprofilaxis en el paciente médico. Estudios randomizados demostraron que esta estrategia disminuye en hasta $60 \%$ la incidencia de eventos tromboembólicos en pacientes mayores de 40 años, hospitalizados por una condición médica aguda y que tienen uno o más factores de riesgo de trombosis ${ }^{41-43}$.

La infección por SARS-CoV-2 aumenta el riesgo de trombosis de forma adicional a los factores de riesgo tradicionales ya comentados. Estudios observacionales reportan incidencias de trombosis sintomática de 25 a $30 \%$ en pacientes en cuidados intensivos por Covid-19, lo que es mayor al 2,7 o $5,5 \%$ reportado en estudios de tromboprofilaxis en paciente médico. En un estudio de 198 pacientes hospitalizados por Covid-19 en Ámsterdam la incidencia de trombosis sintomática a 14 días fue de $3 \%$ en pacientes de sala y $28 \%$ en pacientes de UCI, pese al uso rutinario de HBPM.

En otro estudio de 388 pacientes hospitalizados por Covid-19 en un hospital de Milán, Italia, la incidencia trombosis sintomática fue de $6,6 \%$ en pacientes de sala y de $27 \%$ en pacientes de $\mathrm{UCI}^{44-46}$.

En base a la evidencia descrita las guías de manejo de coagulopatía inducida por Covid-19 recomiendan que todo paciente hospitalizado por esta causa, ya sea en UCI o en sala, incluyendo a mujeres embarazadas e independiente del riesgo de trombosis estimado deben recibir tromboprofilaxis farmacológica ${ }^{47-53}$. La heparina, ya sea HBPM o heparina no fraccionada (HNF) es el fármaco de elección en base a sus efectos pleiotrópicos y posible beneficio en sobrevida en esta población ${ }^{54}$.

La elevada incidencia de eventos trombóticos pese a dosis estándares de tromboprofilaxis en pacientes con Covid-19 junto a la evidencia del posible beneficio de dosis mayores en esta población ha planteado el uso de dosis "intermedias" de heparinas e incluso dosis terapéuticas. El efecto de dosis terapéuticas de anticoagulación en la mortalidad hospitalaria de pacientes Covid-19 fue evaluado en una base de 2.773 pacientes en Nueva York. En pacientes en ventilación mecánica, la mortalidad hospitalaria fue de $29 \%$ con uso de anticoagulación respecto a $62 \%$ con tromboprofilaxis habitual. Este estudio, con limitaciones por su naturaleza observacional, sugiere que la anticoagulación en dosis terapéuticas podría asociarse a una menor mortalidad en pacientes hospitalizados con Covid- $19^{55}$.

Las guías de manejo de la coagulopatía inducida por Covid-19 recomiendan usar dosis estándares de tromboprofilaxis en todo paciente con Covid-19 hospitalizado en sala (enoxaparina $40 \mathrm{mg}$ /día o dalteparina 5.000 U/día o HNF 5.000 $\mathrm{U} \mathrm{c} / 8 \mathrm{~h})^{47-52}$. En pacientes hospitalizados en UCI ciertas guías recomiendan el uso rutinario de dosis intermedias (enoxaparina $40 \mathrm{mg} \mathrm{c} / 12 \mathrm{~h}$ o 0,5 $\mathrm{mg} / \mathrm{kg} \mathrm{c} / 12 \mathrm{~h}$ o HNF $7.500 \mathrm{U} \mathrm{c} / 8 \mathrm{~h})^{18,47}$ mientras que otras guías solo las recomiendan en aquellos pacientes que tienen factores de riesgo adicionales como IMC mayor a 30, múltiples factores de riesgo de trombosis, DD elevado o un score de coagulopatía inducida por sepsis (SIC) elevado ${ }^{48,52}$.

El uso de dosis terapéuticas de anticoagulación, en ausencia de TEV documentado, es decir, como prevención primaria, no está recomendada en base a ausencia de estudios randomizados al respecto.

\section{Tromboprofilaxis extendida en pacientes con Covid-19}

El 60\% de los fenómenos tromboembólicos asociados a una hospitalización ocurren en el 
periodo post alta ${ }^{41}$. El rol de la tromboprofilaxis extendida, bien documentada en ciertos pacientes quirúrgicos, ha sido controversial en el paciente médico debido a un perfil de riesgo desfavorable en los estudios iniciales ${ }^{56-59}$. Sin embargo el análisis posterior de estos datos demostró beneficio en un grupo seleccionado de pacientes; aquellos con bajo riesgo de sangrado, hospitalizados por una condición aguda por más de tres días que al momento del alta tienen movilidad reducida y algún otro factor de riesgo mayor de trombosis como edad sobre 75 años, TEV previa, cáncer, trombofilia, estadía en UCI o DD elevado ${ }^{60,61}$. Para este grupo la FDA aprobó el uso de betrixabán y rivaroxabán como tromboprofilaxis posterior al alta ${ }^{62}$

No hay estudios que evalúen la tromboprofilaxis extendida en pacientes con Covid-19. Considerando la elevada incidencia de trombosis, la estadía prolongada en UCI y el severo desacondicionamiento físico al momento del alta, las guías recomiendan considerar tromboprofilaxis por $2 \mathrm{a}$ 4 semanas desde el egreso hospitalario en aquellos pacientes que estuvieron en UCI o requirieron intubación orotraqueal que tienen movilidad reducida y algún otro factor de riesgo mayor de trombosis al momento del alta ${ }^{48,52}$. Estos son pacientes que cumplen los criterios de inclusión de los estudios que demostraron beneficio de la tromboprofilaxis extendida en el paciente médico.

\section{Conclusiones}

La infección por SARS-CoV-2 es una nueva entidad en la cual el compromiso endotelial y la interacción entre inflamación y trombosis, denominada "respuesta inmuno- trombótica o trombo-inflamatoria", son elementos únicos. El daño endotelial y la intensa respuesta inflamatoria, mediada por citoquinas y leucocitos, determinan una activación global de la coagulación generando un estado protrombótico que lleva a la trombosis masiva de la microcirculación pulmonar y de otros órganos. Este "síndrome microvascular obstructivo" es esencial en el desarrollo de la insuficiencia respiratoria y falla orgánica múltiple. En este contexto, la heparina por sus propiedades anticoagulantes, antiinflamatorias, antivirales y de protección endotelial es el fármaco ideal en esta enfermedad.

En base a la fisiopatología de la enfermedad, a los principios de tromboprofilaxis en paciente médico y a las guías internacionales publicadas, la heparina es parte fundamental del tratamiento de todo paciente hospitalizado con Covid-19.

\section{Referencias}

1. Zhu N, Zhang D, Wang W, Li X, Yang B, Song J, et al. A Novel Coronavirus from Patients with Pneumonia in China, 2019. N Engl J Med 2020; 382 (8): 727-33. doi:10.1056/NEJMoa2001017.

2. Zhang W, Zhao Y, Zhang F, Wang Q, Li T, Liu Z, et al. The use of anti-inflammatory drugs in the treatment of people with severe coronavirus disease 2019 (COVID-19): The experience of clinical immunologists from China, Clinical Immunology (2019), https://doi. org/10.1016/j

3. Berlin DA, Gulick RM, MartÍnez FJ. Severe Covid-19. Published on May 15, 2020, at NEJM.org. DOI: 10.1056/ NEJMcp2009575.

4. Iba T, Levy JH, Levi M, Connors JM, Thachil J. Coagulopathy of Coronavirus Disease 2019. Crit Care Med. 2020 Sep;48(9):1358-1364. doi: 10.1097/ CCM.0000000000004458.

5. Cushman M, Tsai AW, White RH, Heckbert SR, Rosamond WD, Enright P, et al. Deep vein thrombosis and pulmonary embolism in two cohorts: the longitudinal investigation of thromboembolism etiology. Am J Med 2004; 117 (1): 19-25. doi: 10.1016/j.amjmed.2004.01.018. PMID: 15210384.

6. Patel R, Cook DJ, Meade MO, Griffith LE, Mehta G, Rocker GM, et al. Burden of Illness in venous ThromboEmbolism in Critical care: a multicenter observational study. J Crit Care 2005; 20 (4): 341-7.

7. Ackermann M, Verleden SE, Kuehnel M, Haverich A, Welte T, Laenger F, et al. Pulmonary Vascular Endothelialitis, Thrombosis, and Angiogenesis in Covid-19. N Engl J Med 2020; 383 (2): 120-8. doi: 10.1056/NEJMoa2015432.

8. Danzi GB, Loffi M, Galeazzi G, Gherbesi E. Acute pulmonary embolism and COVID-19 pneumonia: a random association? Eur Heart J 2020. https://doi. org/10.1093/eurheartj/ehaa254.

9. Fox S, Akmatbekov A, Harbert J, Li G, Brown J, Vander Heide R. Pulmonary and Cardiac Pathology in Covid-19: The First Autopsy Series from New Orleans 2020. https://doi.org/10.1101/2020.04.06.20050575.

10. Brandon M. Parker, Valerie J. Hart, Rishi Rattan. Coagulopathy in COVID-19: review and recommendations. Division of Trauma Surgery \& Surgical Critical 
Care. DeWitt Daughtry Family Department of Surgery. Leonard M. Miller School of Medicine, University of Miami. https://www.facs.org//media/files/covid19/ umiami_study_uses_of_coagulopathy.ashx

11. Hamming I, Timens W, Bulthuis M, Lely A, Navis G, van Goor H. Tissue distribution of ACE2 protein, the functional receptor for SARS coronavirus. A first step in understanding SARS pathogenesis. J Pathol 2004; 203: 631-7.

12. Gheblawi M, Wang K, Viveiros A, Nguyen Q, Zhong JC, Turner AJ, et al. Angiotensin-Converting Enzyme 2: SARS-CoV-2 Receptor and Regulator of the Renin-Angiotensin System: Celebrating the 20th Anniversary of the Discovery of ACE2. Circ Res. 2020; 126 (10): 145674. DOI: 10.1161/CIRCRESAHA.120.317015.

13. Colafrancesco S, Alessandri C, Conti F, Priori R. COVID-19 gone bad: A new character in the spectrum of the hyperferritinemic syndrome? Autoimmunity Reviews, https://doi.org/10.1016/j.autrev.2020.102573.

14. Dalan R. Commentary. Is DPP4 inhibition a comrade or adversary in COVID-19 infection. diabetes research and clinical practice 164 (2020) 108216.

15. Iacobellis G. Commentary. COVID-19 and diabetes: Can DPP4 inhibition play a role? diabetes research and clinical practice 162 (2020) 108125.

16. Becker RC. COVID19 update: Covid19associated coagulopathy. Journal of Thrombosis and Thrombolysis. https://doi.org/10.1007/s11239-020-02134-3. Published on line: 15 May 2020.

17. Zhang W, Zhao Y, Zhang F, Wang Q, Li T, Liu Z, et al. The use of anti-inflammatory drugs in the treatment of people with severe coronavirus disease 2019 (COVID-19): The Perspectives of clinical immunologists from China. Clin Immunol 2020; 214: 108393. https:// doi.org/10.1016/j.

18. Connors JM, Levy JH. COVID-19 and its implications for thrombosis and anticoagulation. 2020 by The American Society of Hematology. Blood 2020; 135 (23): 2033-40.

19. Joly BS, Siguret V, Veyradier A. Understanding pathophysiology of hemostasis disorders in critically ill patients with COVID-19. Intensive Care Med 2020; 46: 1603-6. https://doi.org/10.1007/s00134-020-06088-1

20. Iba T, Levy JH, Levi M, Thachil J. Coagulopathy in COVID-19. J Thromb Haemost. 2020 Jun 18:10.1111/ jth.14975. doi: 10.1111/jth.14975.

21. Najem MY, Couturaud F, Lemarié CA. Cytokine and chemokine regulation of venous thromboembolism. J Thromb Haemost. 2020 May;18(5):1009-1019. doi: 10.1111/jth.14759.

22. Varga Z, Flammer AJ, Steiger P, Haberecker M, Ander- matt R, Zinkernagel AS, et al. Endothelial cell infection and endotheliitis in COVID-19. The Lancet. https://doi. org/10.1016/S0140-6736(20)30937-5.

23. Thachil J. The versatile heparin in COVID-19. J Thromb Haemost. 2020; 18 (5): 1020-2.

24. Gandhi RT, Lynch JB, Del Rio C. Mild or Moderate Covid-19. N Engl J Med. 2020; 383 (18): 1757-66. doi: 10.1056/NEJMcp2009249.

25. Balasubramanyam M. Does COVID-19 Warn Us to Revisit Virus-Induced Diabetes? Exploratory Research and Hypothesis in Medicine 2020;000(000):000-000. doi: 10.14218/ERHM.2020.00046.

26. Helms J, Kremer S, Merdji H, Clere-Jehl R, Schenck M, Kummerlen C, et al. Neurologic Features in Severe SARS-CoV-2 Infection. N Engl J Med. 2020; 382 (23): 2268-70. doi: 10.1056/NEJMc2008597.

27. Oxley TJ, Mocco J, Majidi S, Kellner CP, Shoirah H, Singh IP, et al. Large-Vessel Stroke as a Presenting Feature of Covid-19 in the Young. N Engl J Med. 2020; 382 (20): e60. doi: 10.1056/NEJMc2009787.

28. Azouz E, Yang S, Monnier-Cholley L, Arrivé L. Systemic arterial thrombosis and acute mesenteric ischemia in a patient with COVID-19. Intensive Care Med 2020; 46 (7): 1464-5. doi: 10.1007/s00134-020-06079-2.

29. Kashi M, Jacquin A, Dakhil B, Zaimi R, Mahé E, Tella $\mathrm{E}$, et al. Severe arterial thrombosis associated with Covid-19 infection. Thromb Res 2020; 192: 75-7.

30. Libby P. The Heart in COVID-19: Primary Target or Secondary Bystander? JACC Basic Transl Sci 2020; 5 (5): 537-42.

31. Ronco C, Reis T, Husain-Syed F. Management of acute kidney injury in patients with COVID-19. Lancet Respir Med 2020; 8 (7): 738-42.

32. Cheng Y, Luo R, Wang K, Zhang M, Wang Z, Dong L, et al. Kidney disease is associated with in-hospital death of patients with COVID-19. Kidney Int 2020; 97 (5): 829-38. doi: 10.1016/j.kint.2020.03.005.

33. Radiological Society of North America. Imaging reveals bowel abnormalities in patients with COVID-19. ScienceDaily. ScienceDaily, 11 May 2020. <www.sciencedaily. com/releases/2020/05/200511142148.htm>.

34. Yang L, Han Y, Nilsson-Payant BE, Gupta V, Wang P, Duan X, et al. A Human Pluripotent Stem Cell-based Platform to Study SARS-CoV-2 Tropism and Model Virus Infection in Human Cells and Organoids. Cell Stem Cell 2020; 27 (1): 125-36.e7. doi:10.1016/j. stem.2020.06.015.

35. Iba T, Levy JH, Warkentin TE, Thachil J, van der Poll T, Levi M; Scientific and Standardization Committee on DIC, and the Scientific and Standardization Committee on Perioperative and Critical Care of the International 
Society on Thrombosis and Haemostasis. Diagnosis and management of sepsis-induced coagulopathy and disseminated intravascular coagulation. J Thromb Haemost 2019; 17 (11): 1989-94.

36. Medcalf RL, Keragala CB, Myles PS. Fibrinolysis and COVID-19: a plasmin paradox. doi: 10.1111/jth.14960.

37. Seitz R, Schramm W. Letter to the editor dic in COVID-19: Implications for prognosis and treatment? J Thromb Haemost 2020; 00: 1-2.

38. Poterucha TJ, Libby P, Goldhaber SZ. More than an anticoagulant: do heparins have direct anti-inflammatory effects? Thromb Haemost. 2017;117(3):437-44.

39. Vicenzi E, Canducci F, Pinna D, Mancini N, Carletti S, Lazzarin A, et al. Coronaviridae and SARS-associated corona virus strain HSR1. Emerg Infect Dis 2004; 10 (3): 413-8.

40. Mycroft-West C, Su D, Elli S, Li Y, Guimond S, Miller $\mathrm{G}$, et al. The 2019 coronavirus (SARS-CoV-2) surface protein (Spike) S1 Receptor Binding Domain undergoes conformational change upon heparin binding. doi: https://doi.org/10.1101/2020.02.29.971093.

41. Schünemann HJ, Cushman M, Burnett AE, Kahn SR, Beyer-Westendorf J, Spencer FA, et al. American Society of Hematology 2018 guidelines for management of venous thromboembolism: prophylaxis for hospitalized and nonhospitalized medical patients. Blood Adv 2018; 2 (22): 3198-225.

42. Turpie AGG. Thrombosis Prophylaxis in the Acutely Ill Medical Patient: Insights from the Prophylaxis in MEDical patients with ENOXaparin (MEDENOX) Trial. Am J Cardiol 2000; 86 (suppl): 48M-52M.

43. Leizorovicz A, Cohen AT, Turpie AG, Olsson CG, Vaitkus PT, Goldhaber SZ; PREVENT Medical Thromboprophylaxis Study Group. Randomized, placebo-controlled trial of dalteparin for the prevention of venous thromboembolism in acutely ill medical patients. Circulation 2004; 110 (7): 874-9.

44. Middeldorp S, Coppens M, van Haaps TF, Foppen M, Vlaar AP, Müller MCA, et al. Incidence of venous thromboembolism in hospitalized patients with COVID-19. J Thromb Haemost 2020; 18 (8): 1995-2002. doi: $10.1111 /$ jth. 14888 .

45. Lodigiani C, Iapichino G, Carenzo L, Cecconi M, Ferrazzi P, Sebastian T, et al; Humanitas COVID-19 Task Force. Venous and arterial thromboembolic complications in COVID-19 patients admitted to an academic hospital in Milan, Italy. Thromb Res 2020; 191: 9-14. doi: 10.1016/j.thromres.2020.04.024.

46. Helms J, Tacquard C, Severac F, Leonard-Lorant I, Ohana M, Delabranche X, et al; CRICS TRIGGERSEP Group (Clinical Research in Intensive Care and Sepsis
Trial Group for Global Evaluation and Research in Sepsis). High risk of thrombosis in patients with severe SARS-CoV-2 infection: a multicenter prospective cohort study. Intensive Care Med. 2020; 46 (6): 1089-98. doi: 10.1007/s00134-020-06062-x.

47. Barnes GD, Burnett A, Allen A, Blumenstein M, Clark NP, Cuker A, et al. Thromboembolism and anticoagulant therapy during the COVID-19 pandemic: interim clinical guidance from the anticoagulation forum. J Thromb Thrombolysis 2020; 50 (1): 72-81. doi: 10.1007/ s11239-020-02138-z.

48. Spyropoulos AC, Levy JH, Ageno W, Connors JM, Hunt BJ, Iba T, et al; Subcommittee on Perioperative, Critical Care Thrombosis, Haemostasis of the Scientific, Standardization Committee of the International Society on Thrombosis and Haemostasis. Scientific and Standardization Committee communication: Clinical guidance on the diagnosis, prevention, and treatment of venous thromboembolism in hospitalized patients with COVID-19. J Thromb Haemost 2020; 18 (8): 1859-65. doi: $10.1111 /$ jth.14929.

49. Thachil J, Tang N, Gando S, Falanga A, Cattaneo M, Levi M, Clark C, Iba T. ISTH interim guidance on recognition and management of coagulopathy in COVID-19. J Thromb Haemost 2020; 18 (5): 1023-6. doi: 10.1111/jth.14810.

50. Baumann Kreuziger L, Lee A, Garcia D, Cuker A, Cushman M, DeSancho M, et al. COVID-19 and VTE/Anticoagulation: Frequently Asked Questions. Version 3.0; last updated May 18, 2020. https://www.hematology. org/covid-19/covid-19-.

51. Sociedad Española de Trombosis y Hemostasia. Recomendaciones sobre profilaxis de enfermedad tromboembolica (ETV) en el embarazo y puerperio durante la pandemia COVID-19. 15 Abril 2020. https:// www.covid-19.seth.es/wp-content/uploads/2020/04/ Recomendaciones-sobre-profilaxis-ETV-en-embarazo-y-puerperio-COVID-19.pdf. [Consultado el 13 de junio de 2020].

52. Marietta M, Ageno W, Artoni A, De Candia E, Gresele P, Marchetti M, et al. COVID-19 and haemostasis: a position paper from Italian Society on Thrombosis and Haemostasis (SISET). Blood Transfus 2020; 18: 167-9. DOI: $10.2450 / 2020.0083-20$.

53. RCOG. Coronavirus (COVID-19) Infection in Pregnancy. Information for healthcare professionals Version 10.1: Published Friday 19 June 2020. https://www.rcog.org.uk/globalassets/documents/ guidelines/2020-06-18-coronavirus-covid-19-infection-in-pregnancy.pdf. [Consultado el 13 de junio de 2020]. 
54. Tang N, Bai H, Chen X, Gong J, Li D, Sun Z. Anticoagulant treatment is associated with decreased mortality in severe coronavirus disease 2019 patients with coagulopathy. J Thromb Haemost 2020; 18 (5): 1094-9. doi: 10.1111/jth.14817.

55. Paranjpe I, Fuster V, Lala A, Russak A, Glicksberg BS, Levin MA, et al. Association of Treatment Dose Anticoagulation with In-Hospital Survival Among Hospitalized Patients with COVID-19. To appear in: Journal of the American College of Cardiology. DOI: https://doi. org/10.1016/j.jacc.2020.05.001.

56. Alexander T. Cohen and Beverley J. Hunt. Is there a role for low-dose DOACs as prophylaxis? Hematology 2019. Hematology Am Soc Hematol Educ Program 2019; 2019 (1): 187-93. doi: 10.1182/hematology.2019000026.

57. Cohen AT, Harrington RA, Goldhaber SZ, Hull RD, Wiens BL, Gold A, et al; APEX Investigators. Extended Thromboprophylaxis with Betrixaban in Acutely Ill Medical Patients. N Engl J Med 2016; 375 (6): 534-44. doi: 10.1056/NEJMoa1601747.

58. Cohen AT, Spiro TE, Büller HR, Haskell L, Hu D, Hull
$\mathrm{R}$, et al; MAGELLAN Investigators. Rivaroxaban for thromboprophylaxis in acutely ill medical patients. $\mathrm{N}$ Engl J Med 2013; 368 (6): 513-23. doi: 10.1056/NEJMoa1111096.

59. Dalen JE, Stein PD, Plitt JL, Jaswal N, Alpert JS. Extended Thromboprophylaxis for Medical Patients. Am J Med 2020; 133 (1): 9-11. doi: 10.1016/j.amjmed.2019.05.050.

60. Chi G, Goldhaber SZ, Kittelson JM, Turpie AGG, Hernández AF, Hull RD, et al. Effect of extended-duration thromboprophylaxis on venous thromboembolism and major bleeding among acutely ill hospitalized medical patients: a bivariate análisis. J Thromb Haemost 2017; 15: 1913-22.

61. Spyropoulos AC, Lipardi C, Xu J, Lu W, Suh E, Yuan Z, Levitan B, et al. Improved Benefit Risk Profile of Rivaroxaban in a Subpopulation of the MAGELLAN Study. Clin Appl Thromb Hemost 2019; 25: 1-9. DOI: $10.1177 / 1076029619886022$.

62. MacDougall K, Spyropoulos AC. New Paradigms of Extended Thromboprophylaxis in Medically Ill Patients. J Clin Med 2020; 9 (4): 1002. doi: 10.3390/jcm9041002. 